\title{
REFLEXIVE HOMOMORPHIC RELATIONS
}

\author{
G.D. Findlay \\ (received December 7, 1959)
}

It is well known that a symmetric and transitive relation on a set is reflexive wherever it is defined. In this note we show that a converse is true for homomorphic relations in certain classes of algebras.

Consider a class $b$ of similar algebras which contains the sub-algebras and quotient algebras of each of its members. Assume also that the direct product $A \times B$ of each pair $A, B$ in $b$ is also an algebra belonging to $b$. The algebras of $b$, being similar, have the same set of operations. We observe that other operations, called compound operations, may be obtained by composition from the assigned operations.

By a homomorphic relation $p$ on an algebra $A$ we mean a subalgebra of the direct product $A \times A$. If the pair $\left(a, a^{\prime}\right) \in P$, we write, as usual, a $p a^{\prime}$.

PROPOSITION. Let the class $b$ have a (possibly compound) ternary operation $f:(x, y, z) \rightarrow f(x, y, z)$ such that

$$
f(x, y, y)=x, \quad f(x, x, y)=y \text {. }
$$

Then a reflexive homomorphic relation $p$ on an algebra $A$ of $b$ is also symmetric and transitive and hence is a congruence on A.

Proof. Let a $\rho a^{\prime}$. Then, since $\rho$ is reflexive, a $\rho$ a and $a^{\prime} p a^{\prime}$. Therefore $f\left(a, a, a^{\prime}\right) p f\left(a, a^{\prime}, a^{\prime}\right)$ so that $a^{\prime} p a$, on account of $(*)$. Hence $\rho$ is symmetric.

This note was written while the author was a Fellow of the Summer Research Institute, Canadian Mathematical Congress.

Can. Math. Bull. vol. 3, no. 2, May 1960 
Again, let $a a^{\prime}$ and $a^{\prime} \rho a^{\prime \prime}$. Then $a^{\prime} \rho a^{\prime}$. Therefore $f\left(a, a^{\prime}, a^{\prime}\right) \rho f\left(a^{\prime}, a^{\prime}, a^{\prime \prime}\right)$ so that a $\rho a^{\prime \prime}$. Hence $\rho$ is transitive.

An example of such a class of algebras is the class of all groups, which includes, of course, the classes of rings and of Boolean algebras, with $f(x, y, z)=x y^{-1} z$.

A discussion of algebras satisfying (*) is contained in [1], where further examples are given.

\section{REFERENCE}

1. J. Lambek, Goursat's theorem and the Zassenhaus lemma, Canad. J. Math. 10 (1957), 45-56.

McGill University 